\title{
BOUNDED AND RELATIVELY BOUNDED SETS
}

\author{
NIEL SHELL
}

All subsets of a field that are intersections of almost-orders of the field are characterised, and all ring topologies on a field which are not finer than any nontrivial locally bounded ring are characterised.

Dürbaum [1] and Kowalsky [2] give conditions which guarantee the intersection of almost-orders is again an almost-order. Dürbaum [1] gives an example of an intersection of almost-orders that is neither an almostorder nor the set $\{0,1,-1\}$. We characterise the subsets of a field that are intersections of almost-orders.

Kowalsky [2] characterises those ring topologies that are a supremum of locally bounded ring topologies. A slight modification of his proof establishes Theorem 2 below in which the ring topologies finer than some proper locally bounded ring topology are characterised.

In this note $K$ denotes a (commutative) field, and, for $D \subseteq K$, we let $D^{*}=\{x \in D: x \neq 0\}$. By a proper topology we mean a topology that is neither trivial nor discrete. Value groups of valuations are written multiplicatively. The supremum (Durchschnitt in [2]) of a collection $T$ of topologies is denoted by $v T$. We refer the reader to [2] for the definition of a bounded set and the definition of a locally

Received 10 February 1987.

Copyright Clearance Centre, Inc. Serial-fee code: 0004-9729/87 $\$ A 2.00+0.00$. 
bounded topology (called a bounded topology in [2]).

By an almost-order (Fastordnung) we mean a subset $A$ of a field $K$ which satisfies

(01) $0,1,-1 \in A ; A \neq K$;

(02) $x, y \in A$ implies $x y \in A$;

(03) each element in $K$ is a quotient of elements in $A$;

(04) there exists $z \in A, z \neq 0$, such that $z(x+y) \in A$ for all $x, y \in A$. (Such an element $z$ will be called a 2-addiator.)

The notion of almost-order is well-known. It is defined here because the definition we have given differs from the one appearing in [2]: Kowalsky omits the requirements $-1 \in A$ and $A \neq K$. With obvious modifications, the results we use from [2] apply to almost-orders as defined here. (We note that if $A$ satisfies (01)-(03) and there exists $w \in K$ such that $w(x+y) \in A$ for all $x, y \in A$, then $A$ satisfies (04)).

The utility of almost-orders is established in [2], where it is shown that, for each almost-order $A$ of a field $K$, the collection $\left\{x A: x \in K^{*}\right\}$ is a neighbourhood base at zero for a ring topology ${ }^{\tau} A$ on $K$ for which $A$ is a $\tau_{A}$-bounded neighbourhood of zero, and, conversely, every proper locally bounded topology is induced by some almost-order in this fashion.

THEOREM 1. A subset $E$ of a field $K$ is con intersection of some collection of almost-orders of $K$ if and only if $0,1,-1 \in E, E$ is a multiplicative semigroup, and $E$ is a bounded subset in some proper locally bounded ring topology on $K$.

Proof. The conditions are obviously necessary. Suppose, on the other hand, they are satisfied. Let $A$ be an almost-order of a topology $\tau_{A}$ in which $E$ is bounded. Since $1 \in E, A \subseteq E A$; so $E A$, which is a product of $\tau_{A}$-bounded sets is a $\tau_{A}$-bounded neighbourhood of zero. We show $E A$ is an almost-order inducing the same topology as $A$. $E A$ satisfies (01) because $E A$ is a bounded and, hence, proper subset of $K$. Obviously $E A$ satisfies (02) and (03). To verify that $E A$ satisfies (04), choose $a / b, a, b \in A^{*}$, such that $(a / b) E \subseteq A$. If $z$ is a 2addiator of $A$, then $z a$ is a 2-addiator of $E A$. 
Next, we observe that, for each $c \in(E A)^{*}, E_{c}=E \cup(c E A)$ is an almost-order. Verification of $(01)-(03)$ is straightforward. We see that $c z$, where $z$ is a 2-addiator of $E A$, is a 2-addiator of $E_{c}$ :

$$
c z\left(E_{c}+E_{c}\right) \subseteq c z(E A+E A) \subseteq c E A \subseteq E_{c} \text {. }
$$

Then $E=n_{c \in(E A)^{* E}}$, and the proof is complete.

The proof above shows that if a set is an intersection of a collection of almost-orders, it is an intersection of a collection of almostorders all of which induce the same topology.

COROLLARY. A subset $E$ of a fiezd $K$ is an intersection of same collection of almost-orders of $K$ if and only if $0,1,-1 \epsilon E, E$ is a multiplicative semigroup, and there is a proper valuation $v$ on $K$ and on element $\gamma \in v\left(K^{*}\right)$ such that $v(x) \leq \gamma$ for alz $x \in E$.

Proof. Each locally bounded topology contains a topology induced by a valuation (see [2]).

We recall that a subset $M$ in a commutative topological ring $(S, \tau)$ is relatively bounded if there is a neighbourhood $V$ of zero such that $V(M \pm M) \subseteq M$. Clearly the empty set and $\{0\}$ are relativey bounded subsets of any topological ring. Any other relatively bounded subset $M$ of a field with a ring topology is a neighbourhood of zero by virtue of the containments

$$
\begin{gathered}
0 \in V(M \pm M) \subseteq M ; \\
V m \subseteq V(M \pm M) \subseteq M, \quad m \in M^{*} .
\end{gathered}
$$

If $M$ and $N$ are relatively bounded, so are $-M$ and $M \cap N$. Therefore each relatively bounded neighbourhood of zero contains a symetric relatively bounded neighbourhood. Certainly the entire ring $S$ is relatively bounded. A relatively bounded set other than the empty set, $\{0\}$ and $S$ will be called proper.

THEOREM 2. A proper ring topology $\tau$ on a field is finer than some proper locally bounded ring topology if and only if there is at least one proper t-relatively bounded subset.

Proof. Suppose there is a relatively bounded neighbourhood of zero $U \neq K$. By the discussion following the definition of relative boundedness, we may assume that $U$ is symetric. We define the set 


$$
A=\{x \in K: x U \subseteq U\},
$$

which was shown in $[2$, p. 179] to be an almost-order and a neighbourhood of zero. Since $A$ is a $\tau$-neighbourhood of zero, $\tau_{A} \leq \tau$ : the $\tau_{A}^{-}$ neighbourhoods $x A, x \in K^{*}$, are $\tau$-neighbourhoods of zero.

Conversely, suppose that there is a proper locally bounded topology $\rho$ weaker than $\tau$. Any $\rho$-bounded neighbourhood of zero $U$ is a proper $\tau$-relatively bounded set: since $(U+U) \cup(U-U)$ is $\rho$-bounded, there is an $\rho$-neighbourhood $V$ of zero (which is therefore a $\tau$-neighbourhood of zero) such that $V(U \pm U) \subseteq U$.

We state formally some definitions for the property "local boundedness" which have obvious analogues for any property.

DEFINITION. A nontrivial ring topology is said to be s-locally bounded ( $n$-locally bounded, z-locally bounded) if it is the supremon of some family of locally bounded ring topologies (respectively, not the supremum of any fonily of locally bounded ring topologies, not finer than any nontrivial locally bounded ring topology).

Note that in the analogues of the above definition only weaker ring topologies are considered.

An $n$-locally bounded topology is sometimes referxed to as being of type $N$. The following four properties are mutually exclusive and each property might be thought of as being "less related" to local boundedness than the one before it:

(1) locally bounded,

(2) s-locally bounded but not locally bounded,

(3) n-locally bounded but not $z$-locally bounded,

(4) z-locally bounded.

EXAMPLE 1: (a) The p-adic topology $\tau_{p}$ on the rationals is locally bounded.

(b) The supremum over all primes $p$ of the topologies $\tau_{p}$ is $s-$ locally bounded but not locally bounded.

(c) If $\tau_{m}$ is a topology defined by the scheme in [3, section 1], then the topology $\tau_{m} \vee \tau_{p}$ is $n$-locally bounded but not $z$-locally bounded.

(d) The topology $\tau_{m}$ described above is $z$-locally bounded. 
Podewski [5] shows each countable field has $2^{2^{c}}$ (where $c$ is the cardinality of the continuum) $n$-locally bounded topologies.

A ring topology on a ring $S$ is $\sigma$-bounded if $S$ is the union of a countable collection of bounded subsets. In [6, proof of Theorem 3] (in [7, Question 2 of Section 2]) it is shown that each proper neighbourhood of zero in a nontrivial $\sigma$-bounded ring topology (ring topology with a nondiscrete countable subset) on a field is also a neighbourhood of zero in some coarser nontrivial first countable ring topology. Thus each nontrivial ring topology which is $\sigma$-bounded or has a nondiscrete countable subset is $s$-first countable.

Thus, all ring topologies on a countable field $K$ are $s$-first countable. Since the assignment $\tau \rightarrow\left\{V_{n}\right\}$, where $\left\{V_{n}\right\}$ is any fixed neighbourhood base at zero for $\tau$, defines an injective map into the set of sequences of subsets of $K$, there are at most $2^{c}$ first countable ring topologies on $K$. Hence, there are $2^{2 c}$ s-first countable, but not first countable, ring topologies on each countable field.

A supremum of uncountably many independent (as defined in [8] or [9]) first countable ring topologies is $s$-first countable but not first countable. If $K$ is an uncountable field, then $K$ is an extension of a subfield $F(x)$, where $F$ is an uncountable subfield and $x$ is transcendental over $F$. Then $\left\{\tau_{x-a}\right\}_{a \in F}$, where $\tau_{x-a}$ is any topology induced by an extension of the $(x-a)$-adic valuation on $F(x)$ to $K$, is an uncountable collection of independent first countable ring topologies. Any valuation-induced topology which is not first countable is $z-$ first countable (because topologies induced by valuations are minimal). EXAMPLE 2. Let $v$ be a valuation on $K$ such that the topology $\tau_{v}$ induced by $v$ is not first countable, and let $w$ be a nontrivial valuationinducing a first countable topology $\tau_{w}$. Then $\tau_{v} \vee \tau_{w}$ is not first countable: The supremum of independent topologies is not discrete, and each sequence of nonzero elements is bounded away from zero in the topology $\tau_{v}$ and, hence, also in the supremum. By construction the supremum is not z-first countable. We do not know whether the supremum is $s$-first countable or $n$-first countable. 


\section{References}

[1] H. Dürbaum, "über die Ganzheitsbereiche bewerteter Korper", Math. 2. 57 (1952), 86-93.

[2] H.J. Kowalsky, "Beiträge zur topologischen Algebra", Math. Nachr. 11 (1954), 143-185.

[3] A.F. Mutylin, "An example of a nontrivial topologization of the field of rational numbers. Complete locally bounded fields", Amer. Math. Soc. Trans2. (2) 73 (1968), 159-179.

[4] L. Nachbin, "On strictly minimal topological division xings", BuZZ. Amer. Math. Soc. 55 (1949), 1128-1136.

[5] K.P. Podewski, "The number of field topologies on countable fields", Proc. Amer. Math. Soc. 39 (1973), 33-38.

[6] N. Shell, "Maximal and minimal ring topologies", Proc. Amer. Math. Soc. 68 (1978), 23-26.

[7] N. Shell, "Connected and disconnected fields", Topology App Z. (to appear).

[8] H. Weber, "Unabhängige Topologien, zerlegung von Ringtopologien", Math. 2. 180 (1982), 379-393.

[9] w. Więs Law, "Independent topologies on fields", Comment. Math. Univ. St. Pcal 26 (1977), 201-208.

The City College of New York (CUNY)

Convent Avenue at 138th street

New York, NY 10031, USA 